\title{
The Mission Planning Lab: A Visualization and Analysis Tool
}

\author{
Sarah C. Daugherty, Benjamin W. Cervantes \\ Wallops Systems Software Engineering Branch \\ NASA Wallops Flight Facility \\ Wallops Island, VA, USA \\ Sarah.C.Daugherty@nasa.gov, Benjamin.W.Cervantes@nasa.gov
}

\begin{abstract}
Simulation and visualization are powerful decision making tools that are time-saving and cost-effective. Space missions pose testing and evaluation challenges that can be overcome through modeling, simulation, and visualization of mission parameters. The National Aeronautics and Space Administration's (NASA) Wallops Flight Facility (WFF) capitalizes on the benefits of modeling, simulation, and visualization tools through a project initiative called The Mission Planning Lab (MPL).
\end{abstract}

Keywords-modeling, simulation, software integration, visualization

\section{INTRODUCTION}

The Mission Planning Lab (MPL) is a project designed and developed by the NASA Wallops Systems Software Engineering Branch. The purpose of MPL is to use simulation and visualization to aid in the planning and decision making processes of a mission project. MPL is a collection of commercial-off-the-shelf, government-off-theshelf, and custom software. The commercial software packages that MPL utilizes are Satellite Toolkit (STK), MatLab, ArcGIS, and MS Visual Studio. MPL provides a mathematically correct, visually rich environment that allows realistic simulation, presentation and evaluation of platform selection, flight profiles, and range asset placement. By integrating detailed information on vehicle capabilities, range capabilities, and mission specific objectives, MPL meets several critical needs for the Wallops Research Range, including accurate and timely decisions about vehicle trajectories, attitude maneuvers, and ground range asset placement in order to successfully meet mission objectives.

\section{METHODS}

The goals of MPL are accomplished through four main techniques: visibility, feasibility, variability, and certainty.

\section{A. Visibilty}

MPL provides visibility for a mission by representing a variety of mission aspects in visually-rich $2 \mathrm{D}$ and $3 \mathrm{D}$ environments. Mission aspects that can be visualized in MPL include dynamic, detailed 3D models, Geographic Information Systems (GIS) data including high resolution imagery, terrain, and population data, dynamic spacecraft and launch vehicle trajectory and attitude data, magnetic field modeling, radar coverage, and line of sight (Fig. 1).

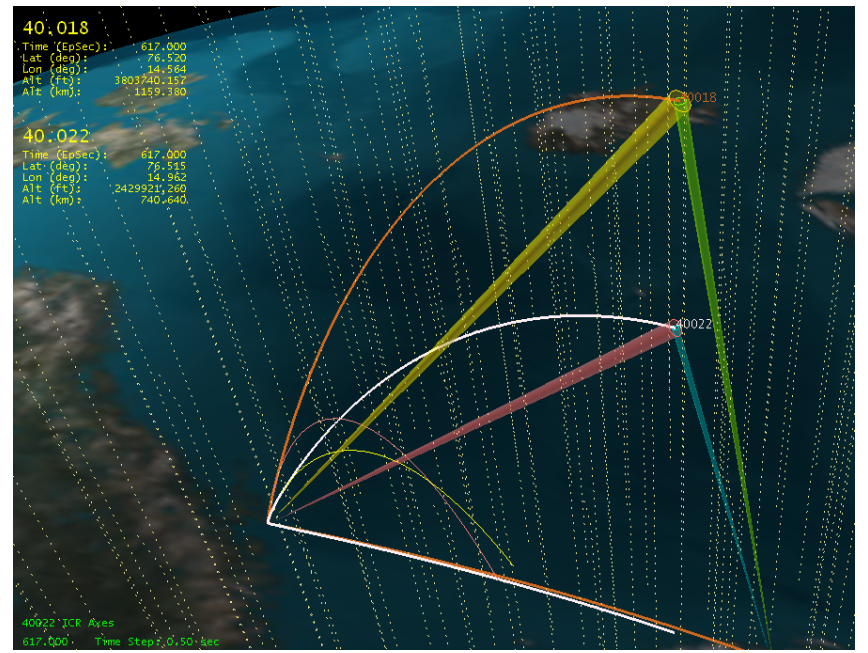

Figure 1. This image shows how the entire Kletzing/TRICE mission looked after it occurred. Two launch vehicles (orange line and white line) were launched within two minutes of each other to study the magnetic field around the North Pole. Magnetic field lines are shown as dotted yellow lines and radar coverage as yellow, red, green, and blue shaded cones.

Dynamic, detailed 3D models provide engineers and scientists with the ability to see launch vehicle payloads,

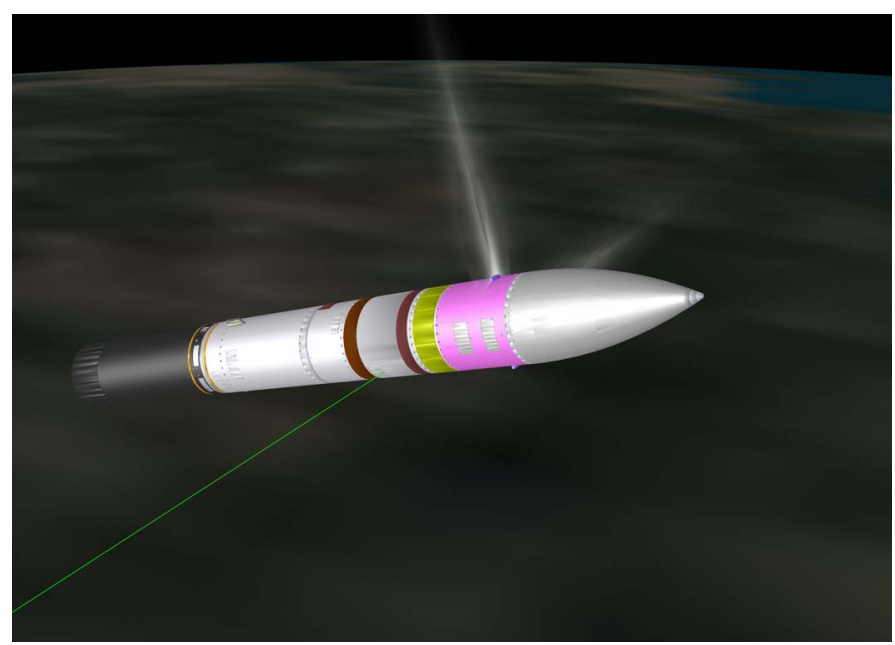

Figure 2. Attitude Control System (ACS) thrusters firing on a 3D detailed model of launch vehicle and payload. Simulation of the thrusters aids in understanding the attitude manuevers performed. In this figure, the thrusters are turning the vehicle perpendicular to the velocity vector (green line). 


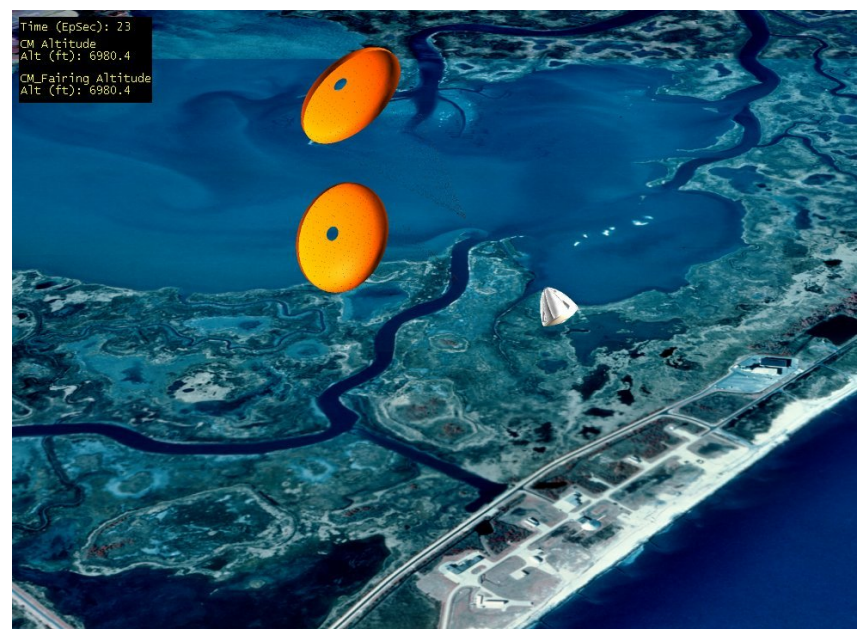

Figure 3. 3D models can show stage separation events, including motor separations, fairing separations, and parachute deployments (seen above).

sensor locations, and stage separation events (Figs. 2, 3). Using 3D models in combination with vehicle orientation yields a wide variety of analysis capabilities such as reports on solar sensor time in sun and depictions of the field of view of telescopes or other on-board instruments.

GIS data provides visibility to safety personnel and mission managers regarding the effects of a mission on the surrounding area. MPL supports missions that occur in a variety of locations around the globe, from the mountains of Alaska and Norway to the east coast of the United States, which require a wide range of imagery, elevation, and population data. MPL acquires this data from the NASA Wallops Flight Facility's GIS database, as well as the US Geological Survey.

GIS data includes high resolution imagery, terrain/elevation data, and population density data. High resolution imagery (Fig. 4) illustrates the location of high value assets to be protected, such as buildings and roads, adjacent to launch and impact areas.

Including terrain, or elevation, data in MPL simulations and visualizations increases analysis capabilities for radar coverage around terrain obstacles, such as mountains (Figs.

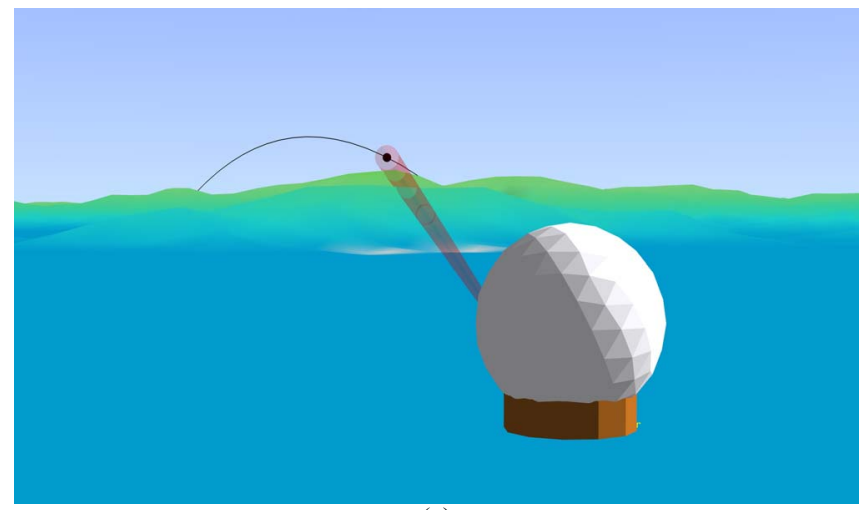

(a)

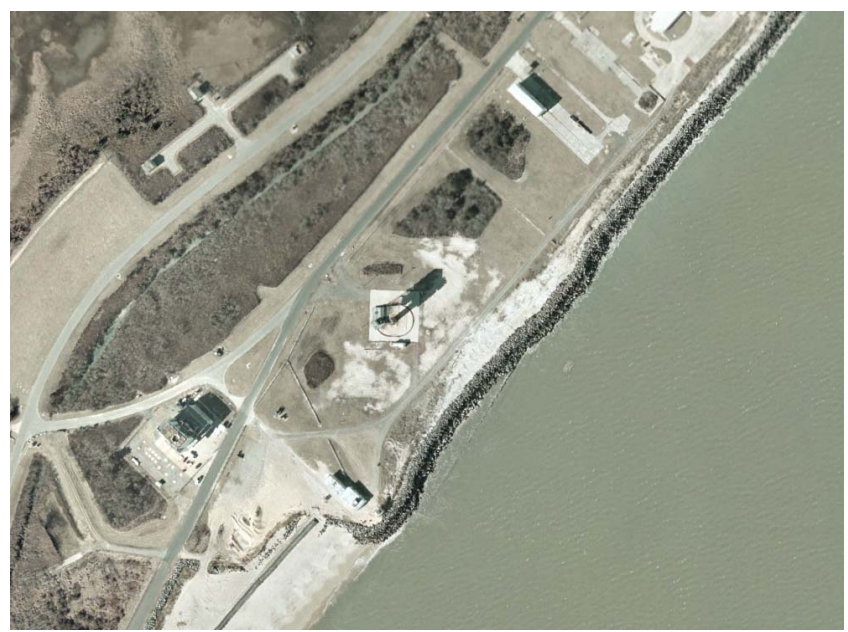

Figure 4. High resolution imagery of NASA WFF launch pad and surrounding area.

5,6). For an accurate radar link budget report, containing line of sight time durations and signal strength among other parameters, it is necessary to import terrain data into a simulation scenario and force radars to abide by the masking rules the terrain presents.

MPL has the capability to ingest some types of raw GIS data files directly or interface with ESRI's GIS software ArcGIS. The interface with ArcGIS allows information such as blast zones, road blocks, and population centers to be overlaid on the already included map imagery and/or terrain (Fig. 7). One of the advantages of overlaying GIS information using ArcGIS in a simulation scenario is maintaining a manageable file size. The MPL ArcGIS interface allows the scenario to reference the location of the GIS data on a server for overlays. Conversely, including each GIS data file can exponentially increase the file size and disk space required for a simulation or visualization, preventing portability and usability.

\section{B. Feasibility}

MPL provides mission decision makers with the knowledge to determine if a mission can be accomplished.

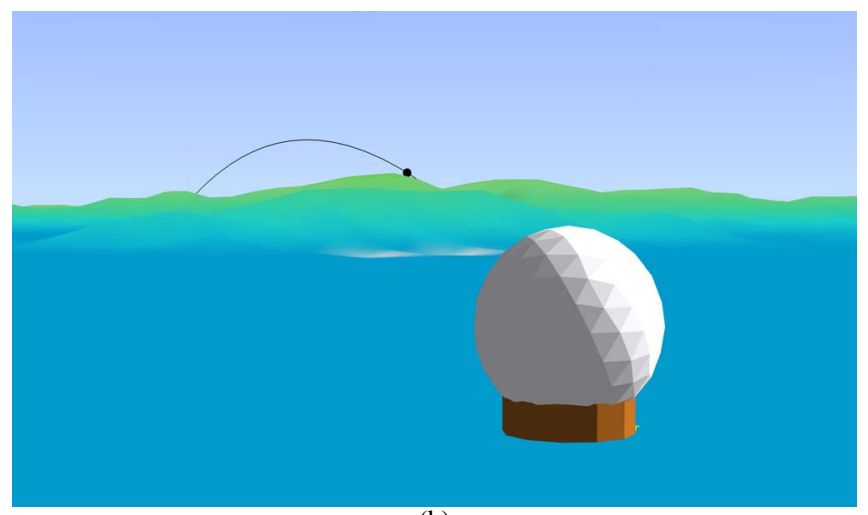

(b)

Figure 5. Terrain data for a mobile radar located on Antigua. (a) The red cone shows the radar line of sight tracking a launch vehicle (black dot) over the mountain range in the distance. (b) A few seconds further down the trajectory, the radar loses sight of the vehicle as it drops below the horizon created by the mountain range. 


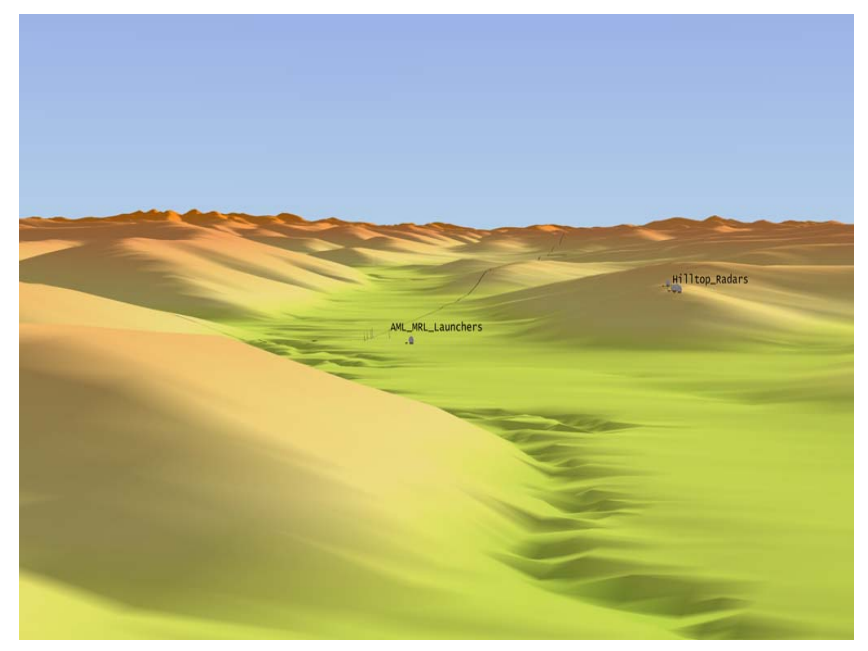

Figure 6. Terrain data for Poker Flats Research Range in Alaska. The launch pads are located in the green valley and the radars are located on top of the hill (right side of picture). The color scale shows relative elevations from low (light green/yellow) to high (orange/red); color scheme is configurable.

MPL reflects mission feasibility through ground and spacebased assets, tracking line of sight to spacecraft or launch vehicles, link margin analysis reports, launch time determination for sunlight requirements, and trajectory formulation to meet mission success criteria.

Ground based assets that MPL includes are radar tracking stations, telemetry transmitting and receiving stations, and camera optics stations. Using basic knowledge of these stations, such as latitude, longitude, and altitude, allows MPL to calculate line of sight from the ground station to the launch vehicle or spacecraft. The line of sight can be displayed visually, as 3D graphics (Fig. 8), or analytically, as

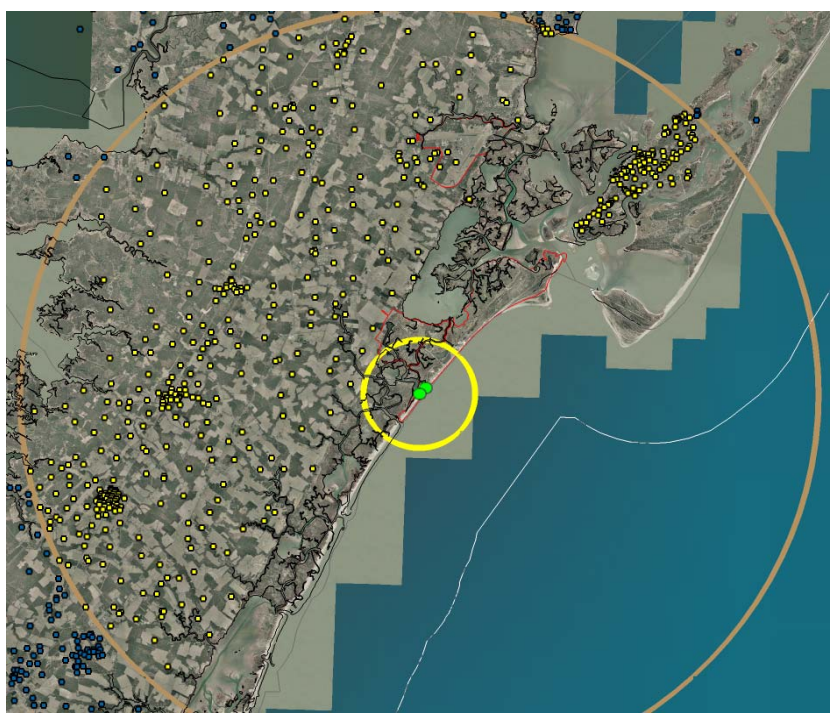

Figure 7. ArcGIS project information overlaid on map imagery in MPL simulation. Green dots are launch pads, yellow circle is $10 \mathrm{~nm}$ radius from pads, brown circle is $30 \mathrm{~nm}$ radius from pads, yellow dots are population centers (houses, businesses, etc.) inside the $30 \mathrm{~nm}$ radius, blue dots are population centers outside the $30 \mathrm{~nm}$ raduis, and the red line encircles NASA Wallops Flight Facility property.

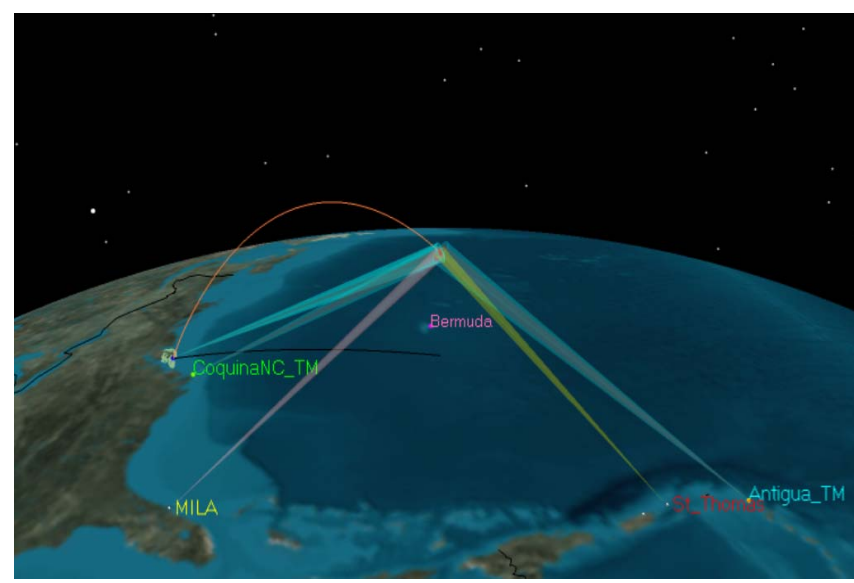

Figure 8. Line of sight calculated to a launch vehicle (orange trajectory line) from different ground assets (radar tracking and telemetry stations, shown as colored cones pointing to the current launch vehicle position).

a report file containing data columns. The line of sight report contains information about the start, stop, and duration times of when the vehicle is visible to the ground station, not beyond the horizon.

Line of sight is important for formulating a trajectory to meet mission requirements, such as a specific altitude for payload experiments while maintaining contact with a ground station. Particularly, WFF Range Safety Officers are interested in this constant communication with the launch vehicle and payload for positional data in order to keep high value assets, people and property, safe. Space based assets that MPL includes are networks of communications systems, such as the Deep Space Network (DSN) and the Tracking and Data Relay Satellite System (TDRSS) (Fig. 9).

For users who need more comprehensive analysis than fundamental line of sight reports, MPL-generated link margin reports provide a better understanding of communication links between launch vehicles, payloads/experiments, and ground stations. Based on vehicle trajectory and attitude, and taking into consideration plume attenuation and ground asset capabilities such as beam

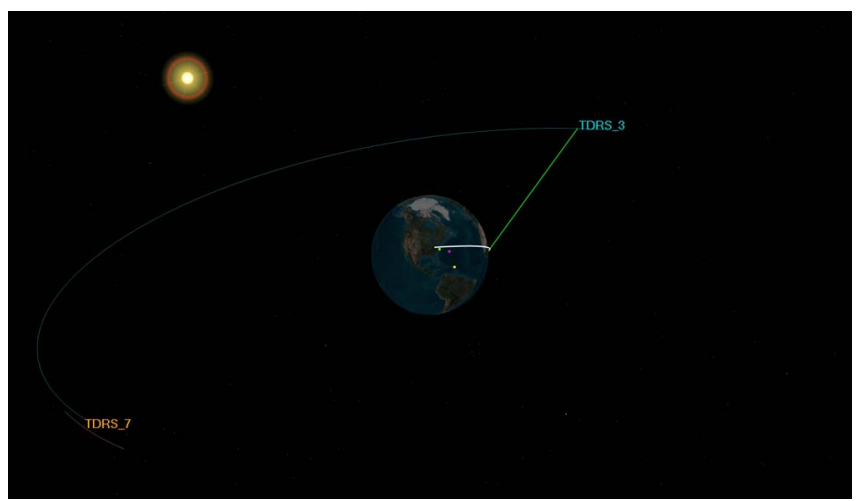

Figure 9. TDRSS 7 and TDRSS 3 satellites (orange and blue labels) tracking a vehicle launch from WFF (white line) orbiting the Earth. The line of sight from the vehicle's position to TDRSS is shown in green.

Depending on the location of the vehicle and TDRSS around the Earth, a TDRSS satellite might not be able to see the launch vehicle, as is the case with TDRSS 7. 


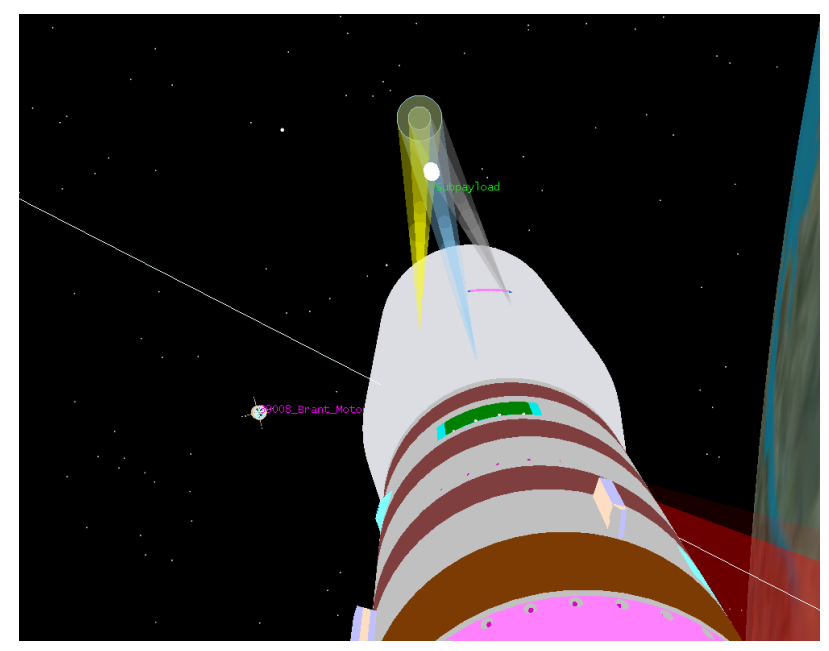

Figure 10. In this mission, an objective was to perform a payload attitude maneuver to focus the on-board telescopes (yellow, blue, and gray cones) from the previously ejected vehicle motor (purple label) to the subpayload (white cylinder with green label). MPL was able to show mission managers and engineers that the current trajectory and attitude configuration would meet this mission objective.

width and data rate, a link margin analysis determines look angles and signal strength. Link margin reports can be produced for a variety of different methods of communication: radar tracking, telemetry data relay, and telemetry commanding.

In addition to line of sight and link analysis reports, which have to do primarily with communications, feasibility may be desired using different aspects of a mission. One example is trajectory or attitude formulation to meet mission requirements. MPL can dynamically display current trajectory and attitude configurations for flight engineers to assess position and maneuvers for specific mission objectives (Fig. 10). If the current configuration shows that the objectives are not met, another iteration of trajectory and attitude can be created and displayed.

Another example to demonstrate the feasibility of a mission is to use MPL to display solar aspect angles to determine an optimum launch time for payload experiments

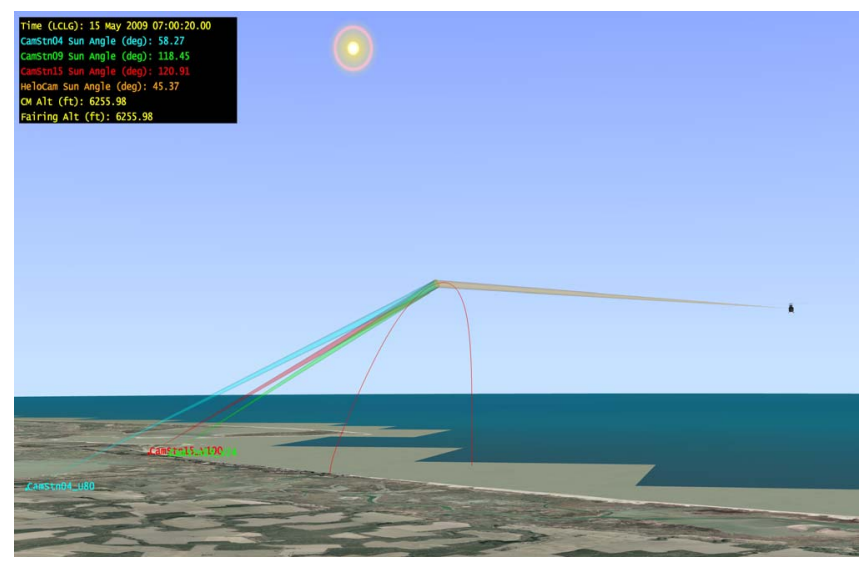

Figure 11. Ground camera stations (side left) and a helicopter camera station (side right) with targeted coverage on a launch vehicle. The upper left corner displays sun angles to each camera station. and optical camera station tracking. Payload experiments range from requiring abundant sunlight to hardly any sunlight. Optical camera stations tracking the vehicle require no direct sunlight pointing into the camera lens as well as no backlighting. The combination of requirements produced poses a unique problem which MPL can solve by varying the time of day and analyzing the solar aspect angles formed relative to vehicle/payload and camera location (Fig. 11).

\section{Variability}

MPL provides the ability to adjust various vehicle and mission characteristics to improve mission performance or success. As a visualization tool, MPL can display multiple trajectories and attitudes for comparison in conjunction with other mission parameters such as mobile telemetry and radar assets (Fig. 12). MPL also has the ability to vary onboard sensor locations and launch time/date for scheduling purposes. Simulating the variability of a mission allows customers to make cost benefit or mission assurance decisions.

Variability is directly connected to feasibility, discussed in the previous section. When assessing a mission's feasibility, if certain criteria are not met by the current mission design, mission parameters can be varied to meet the criteria. For example, examine a mission with trade-offs in ground station locations for line of sight to the launch vehicle payload. Assume the minimum success criteria for a payload experiment is 60 seconds of data downlink to a ground station. If the current nominal trajectory only provides 45 seconds of data, determined in the feasibility process by an MPL line of sight or link margin analysis report, the variability process can begin by adjusting trajectory parameters to give the ground station increased access time to the payload (Fig. 13).

Another solution is to identify other geographic locations that are included in WFF ground asset sites for mobile radar and telemetry. MPL has electronically cataloged all WFF range assets and parameters to be incorporated into a simulation scenario quickly and easily, streamlining the

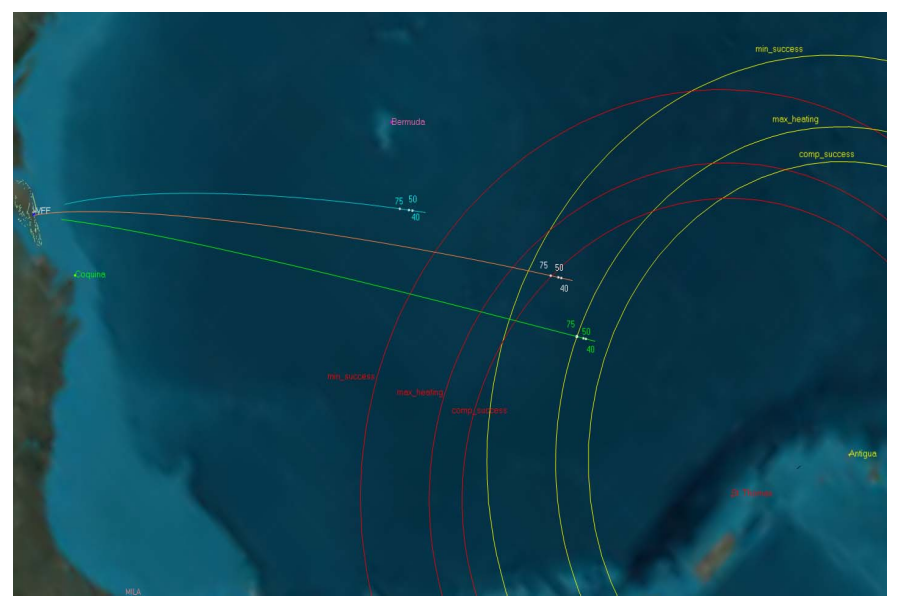

Figure 12. Multiple vehicle trajectories (blue, orange, and green lines) yield different levels of mission success, represented by red and yellow range rings circling around two different WFF ground station assets, Antigua and St. Thomas. 


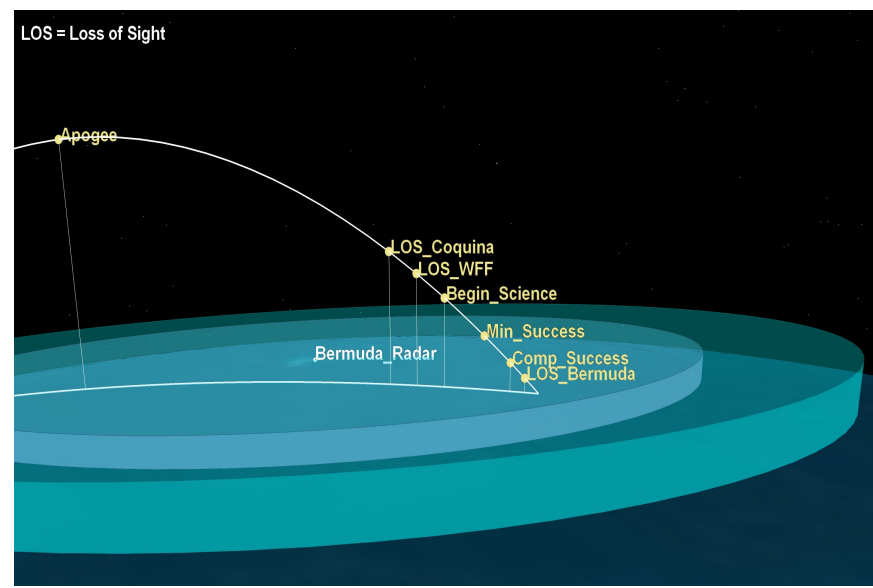

Figure 13. Vehicle trajectory (white line) marked with different mission objectives (yellow dots and labels). The inner blue ring has a height that corresponds to the minimum success criteria for the payload science experiment; the outer blue ring has a height that corresponds to the comprehensive success criteria for the payload.

feasibility-variability process.

\section{Certainty}

MPL can represent the accuracy of calculated mission parameters, through its sophisticated 3D graphics and/or detailed output reports and graphs. The most valuable aspect of MPL verifying and validating mission certainty is in comparing pre-flight results of MPL with post-flight analysis incorporated in MPL.

MPL reflects the accurate analysis of many mission parameters in a single simulation scenario. Continually logging occasions when MPL simulations match pre-flight or post-flight analysis results obtained by specialized engineers give MPL its well-known credibility and prove its reliability. Elements contained in the verification and validation log span a variety of topics such as accurate yaw, pitch, and roll rates, look angles from ground stations to vehicles, on-board telescope field of view and pointing, calculated optimum launch window, calculated satellite lifetime decay, and magnetic field vector locations (Figs. 14, 15).

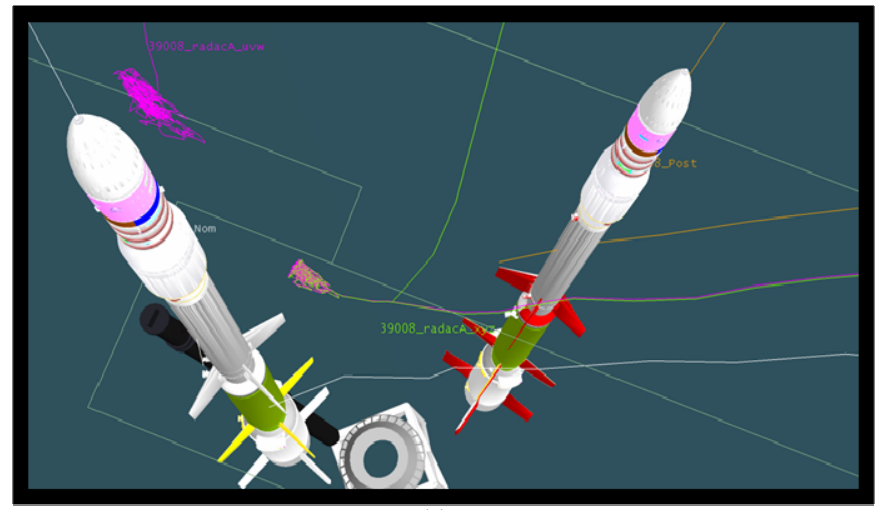

(a)

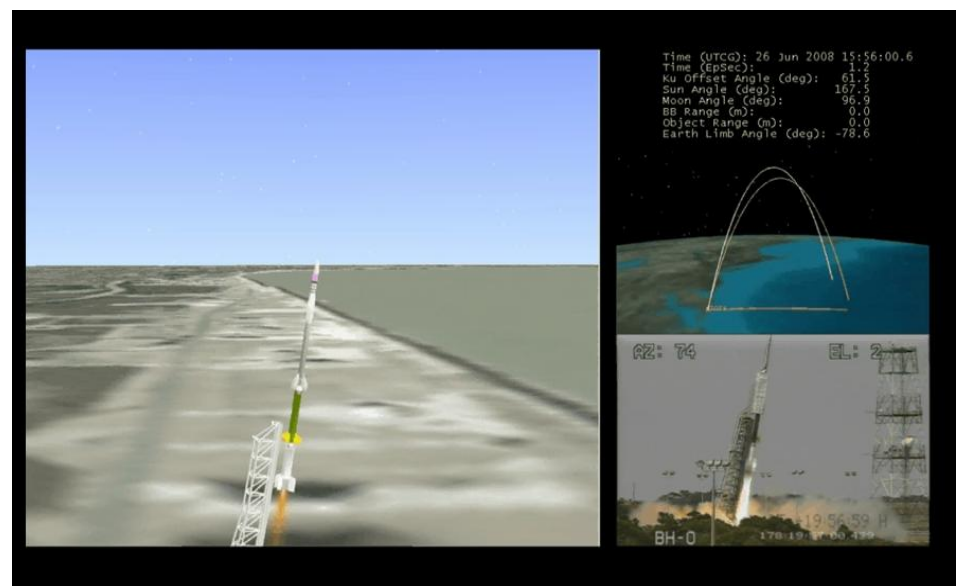

Figure 14. MPL simulated mission view (left large view and right uppr view) versus the actual mission view (right lower view) obtained from optical camera stations.

\section{RESULTS}

To increase flexibility and ease of customer service, MPL produces a variety of deliverables that are versatile and mobile. The deliverables range from an entire simulation and visualization environment to text file reports of specific mission parameters. MPL provides pre-flight and post-flight mission analysis, mission visualization, radar link analysis, and custom reports and graphs.

At the lowest level of detail and capability, MPL has enabled the ability to export vehicle trajectory and other simulation object elements to be viewed in Google Earth (Fig. 16). This is accomplished by creating KML files from the simulation parameters, which can be opened in conjunction with the GIS data and map resources of Google Earth to show 3D images of flight path, one of the advantages of this MPL product. Another advantage of KML files is quick delivery to the customer; MPL has an automated utility which reads simulation data and writes KML files.

Slightly increasing the level of detail and capability,

\begin{tabular}{|c|c|c|c|c|c|}
\hline \multicolumn{3}{|c|}{ LaunchVehicle-39008_Post: } & \multicolumn{2}{|c|}{ This report contains positio } & \multirow{2}{*}{$\begin{array}{l}\text { difference } \\
\text { Range (km) }\end{array}$} \\
\hline Time & (EpSec) & $\mathrm{x}(\mathrm{km})$ & $y(\mathrm{~km})$ & $\mathrm{z}(\mathrm{km})$ & \\
\hline & 0.500 & -0.007303 & -0.014048 & 0.007618 & 0.017570 \\
\hline & 0.700 & -0.006997 & -0.014416 & 0.008352 & 0.018070 \\
\hline & 0.900 & -0.008595 & -0.013067 & 0.007754 & 0.017457 \\
\hline & 1.100 & -0.008055 & -0.016215 & 0.009673 & 0.020527 \\
\hline & 1.320 & -0.010752 & -0.016267 & 0.010337 & 0.022070 \\
\hline & 1.520 & -0.012526 & -0.015257 & 0.009188 & 0.021774 \\
\hline & 1.720 & -0.013238 & -0.018549 & 0.011412 & 0.025486 \\
\hline & 1.920 & -0.014968 & -0.020898 & 0.013059 & 0.028833 \\
\hline & 2.120 & -0.018146 & -0.018680 & 0.011169 & 0.028337 \\
\hline & 2.320 & -0.019987 & -0.021474 & 0.012505 & 0.031891 \\
\hline & 2.520 & -0.022935 & -0.021328 & 0.012975 & 0.033901 \\
\hline & 2.720 & -0.026667 & -0.020317 & 0.011270 & 0.035368 \\
\hline & 2.920 & -0.027711 & -0.026226 & 0.015422 & 0.041153 \\
\hline & 3.120 & -0.029942 & -0.030260 & 0.019783 & 0.046942 \\
\hline & 3.320 & -0.033939 & -0.027341 & 0.016512 & 0.046605 \\
\hline & 3.520 & -0.036626 & -0.028750 & 0.018223 & 0.050001 \\
\hline & 3.720 & -0.037835 & -0.034627 & 0.023817 & 0.056549 \\
\hline & 3.920 & -0.039224 & -0.041506 & 0.028973 & 0.064037 \\
\hline & 4.120 & -0.043179 & -0.039130 & 0.027315 & 0.064356 \\
\hline & 4.320 & -0.047746 & -0.036002 & 0.025293 & 0.064927 \\
\hline
\end{tabular}

(b)

Figure 15. (a) Nomial pre-flight trajectory and attitude (left vehicle) versus actual post-flight trajectory and attitude (red-finned vehicle). (b) Detailed report of the positional differences between nominal and actual. The difference in position at launch, as seen in (a) are due to wind weighting applied at launch. 


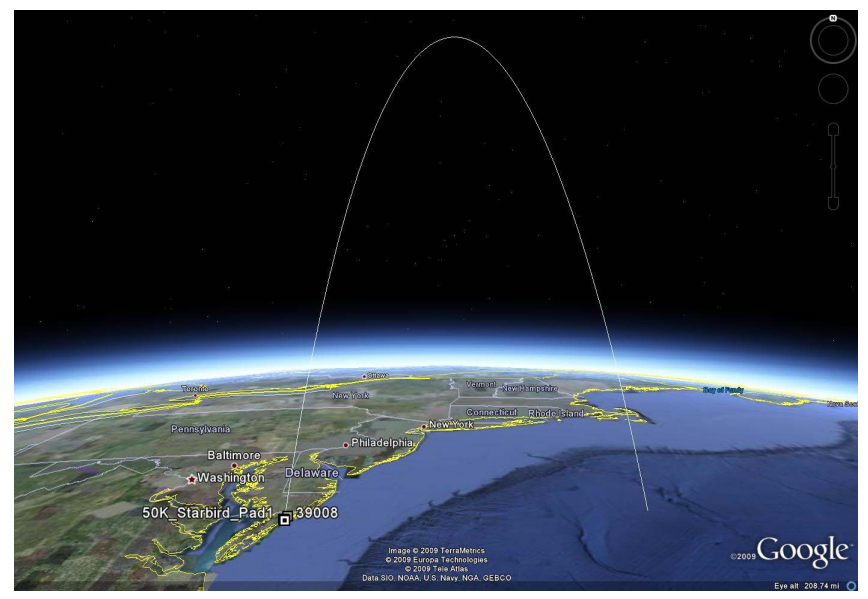

Figure 16. Launch vehicle trajectory displayed in Google Earth.

MPL can provide users with snapshots or images of the 3D and/or 2D simulated animation. These snapshots can include report parameters, such as time, altitude, and sun angle, but are limited in the fact that the image is static. Snapshot images only provide a fixed view of mission details, but can be created quickly for urgent customer needs (Fig. 1). The snapshots can be of almost any image file type, .jpg, .tif, .png, etc, and the size of the file is accordingly dependent.

At the next highest level of detail and capability is the media file. MPL can create and record media files, such as AVI or WMV, to play on any computer or media player. These files allow visualization of the mission in timedynamic 3D animation and viewing from multiple perspectives. Media files can also present reports and graphs, but are limited in detail to the capacity of dynamic information that a human can see in a single display. Media files are much slower in delivery than snapshots images and KML files, as they require much more time to orchestrate. Another drawback to media files are large file sizes that can result from incorporating a large number of analytical data, such as GIS, reports, and multiple view perspectives.

The MPL product at the very highest level of detail and capability is a bundled simulation environment. With this environment, users can visualize a mission from any desired perspective in time-dynamic 3D animation, as well as access detailed reports and graphs at the click of a button. This file includes many mission details, such as radar coverage, link analysis, 3D vehicle and payload models, and measurements. The file gives the user nearly full control of the mission simulation output; maintaining the capability to interface with ArcGIS, allowing the user to import and overlay any GIS data desired, as well as the ability to create their own snapshot images and media files of the 3D and/or 2D visualization window. The only shortcomings to the bundled simulation environment are it requires a specialized viewer application to open and the file size can become large quickly depending on the data included.

\section{CONCLUSION}

Missions supported by MPL have seen many benefits due to rapid mission prototyping and visual capabilities. Current capabilities of MPL are:

- Ingest, generate vehicle and spacecraft trajectories.
- Incorporate vehicle and spacecraft attitude maneuvers.

- Utilize detailed 3D mechanical models.

- Depict stage separation events.

- Illustrate payload deployment.

- Simulate line of sight and link margin analysis for ground and space based assets.

- Display atmospheric layers.

- Depict terrain with GIS.

- Simulate ocean vessel traffic near launch locations for range safety.

- Automatic report and graph generation.

- Mixed media - integrating KML files, live video, and simulation scenarios into a single MPL product.

MPL allows project managers, engineers, and scientists to identify potential risks and issues early in the project development cycle, saving time and money. Post mission modeling through MPL also offers benefits to investigators of mission anomalies by showing customers what actually happened in a powerful visualization environment enhanced with reports and graphs.

\section{FUTURE WORK}

Despite the advanced simulation, visualization, and analysis abilities that MPL currently has, unused resources and capabilities remain which will increase the visibility, feasibility, variability, and certainty of MPL. The following are some areas of research and development for the near future:

- Optimize range safety tools.

- Build a suite of rocket motor models.

- Provide products which display on Science on a Sphere.

- Incorporate magnetic field model.

- Capitalize on interface with MATLAB.

- Remodel plume attenuation using actual post-flight data.

- Utilize a wider variety of reports and graphs.

- Increase efficiency of ocean vessel traffic modeling.

- Expand capabilities beyond launch vehicles and spacecraft to Uninhabited Aerial Vehicles (UAVs) and scientific balloons.

MPL has also yielded a real-time spin-off project called Visualization in Real Time Experiment (VIRTEx). In this project, the same simulation environment MPL customers are accustomed to viewing can be seen during real-time flight operations. The VIRTEx environment uses the same detailed 3D mechanical models and dynamic reports utilized by MPL. VIRTEx displays vehicle attitude maneuvers, stage separation events, and dynamic alphanumeric reports on mission parameters as data enters the WFF Range Control Center during powered vehicle flight.

\section{ACKNOWLEDGEMENT}

S.C.D and B.W.C thank Jay Pittman, Chief of NASA WFF Range and Mission Management Office, and the managers, scientists, and engineers of the NASA WFF Applied Engineering and Technology Directorate (AETD), 
the Sounding Rocket Program Office (SRPO), and the NASA Sounding Rocket Operations Contract (NSROC). 